\title{
A GCH EXAMPLE OF AN ORDINAL GRAPH WITH NO INFINITE PATH
}

\author{
JEAN A. LARSON
}

\begin{abstract}
It is hard to find nontrivial positive partition relations which hold for many ordinals in ordinary set theory, or even ordinary set theory with the additional assumption of the Generalized Continuum Hypothesis. Erdös, Hajnal and Milner have proved that limit ordinals $\alpha<\omega_{1}^{\omega+2}$ satisfy a positive partition relation that can be expressed in graph theoretic terms. In symbols one writes $\alpha \rightarrow(\alpha \text {, infinite path })^{2}$ to mean that every graph on an ordinal $\alpha$ either has a subset order isomorphic to $\alpha$ in which no two points are joined by an edge or has an infinite path. This positive result generalizes to ordinals of cardinality $\aleph_{m}$ for $m$ a natural number. However, the argument, based on a set mapping theorem, works only on the initial segment of the limit ordinals of cardinality $\aleph_{m}$ for which the set mapping theorem is true. In this paper, the Generalized Continuum Hypothesis is used to construct counterexamples for a cofinal set of ordinals of cardinality $\aleph_{m}$, where $m$ is a natural number at least two.
\end{abstract}

1. Introduction. Erdös, Hajnal and Milner [3] were the first to look at the partition relation $\alpha \rightarrow(\alpha \text {, infinite path })^{2}$ in their 1969 paper on set mappings. They were able to prove positive results for many ordinals, but their method using set mappings only worked for ordinals of relatively simple order structure. However, this particular relation looked most promising as a nontrivial one that many ordinals might satisfy. J. Larson [6] used Martin's Axiom to extend the positive result to limit ordinals less than the continuum. (It is not hard to construct counterexamples for sucessor ordinals.)

However, under the assumption of the Generalized Continuum Hypothesis, above the continuum, counterexamples start to appear, as indicated in the statement of the main theorem below.

THEOREM. Assume the Generalized Continuum Hypothesis. For every positive integer $n \geq 2$, there is a cofinal set of ordinals $\alpha<\omega_{n}$ so that

$$
\alpha \nrightarrow(\alpha, \text { infinite path })^{2} .
$$

For ordinals of power continuum, the situation appears to be more delicate. In A diamond example of an ordinal graph with no infinite paths, J. Baumgartner and J. Larson [1] used Jensen's Diamond Principle to show that for all ordinals $\alpha$ with $\omega_{1}^{\omega+2} \leq \alpha<\omega_{2}$, the negative partition relation $\alpha \nrightarrow(\alpha \text {, infinite path })^{2}$ holds.

Received by the editors October 13, 1986.

1980 Mathematics Subject Classification (1985 Revision). Primary 03E50, 04A20, 05A05; Secondary $05 \mathrm{~A} 17$.

Research partially supported by National Science Foundation Grant DMS-8600931 and by P. Erdös. 
Recall that $\alpha \nrightarrow(\alpha \text {, infinite path })^{2}$ means that there is a graph on $\alpha$ which has no independent (edge-free) subsets of order type $\alpha$ and no infinite path. An infinite path is an infinite sequence $v_{0}, v_{1}, \ldots$ of vertices (no repeats allowed), so that for all $i, v_{i}$ is joined by an edge to $v_{i+1}$.

The basic counterexamples are constructed as the comparability graphs of trees. The trees are constructed so that they have no infinite branches. This constraint guarantees that the comparability graphs have no infinite paths. The weak form of the Diamond Principle is used to guarantee that there are no large independent subsets.

Rather than work with the ordinals directly, it is more convenient to work with a richer structure based on finite sequences of ordinals. The ordering of any ordinal can be imitated by the lexicographic ordering on a suitably chosen set of finite sequences of ordinals. Such a representation does not conveniently provide all the information desired for building the counterexample, so products of such sequences are used to build the richest counterexample in the paper. In $\S 2$, a relatively simple counterexample is constructed to give the basic ideas of the proof without too many complicating details. The richest counterexample is given in $\S 3$. In $\S 4$ the counterexamples are lifted to ordinals via pinning to prove the theorem quoted above.

The remainder of this section is devoted to a review of some basic definitions, terminology and well-known facts. In general our set theoretic notation is standard, and T. Jech's book Set theory [5] may be used as a reference.

Order types, that is order-isomorphism types, are partially ordered by embeddability. An order type $\phi$ is indecomposable if whenever $\phi=\pi+\theta$, then $\phi \leq \pi$ or $\phi \leq \theta$. We are particularly interested in the order types of ordinals, converse ordinals and their products. If $\phi$ is linearly ordered by $<$, then the converse $\phi^{*}$, is ordered by $<^{*}$, where $x<^{*} y$ if and only if $y<x$.

A bounded subset $S$ of an ordinal $\alpha$ is one such that $\sup (S)<\alpha$. An unbounded subset of $\alpha$ is one which is not bounded. A closed subset $C$ of $\alpha$ is a subset such that $\sup (S)$ is in $C$ for all bounded subsets $S$ of $C$. A stationary subset $S$ of $\alpha$ is a subset such that the intersection $C \cap S$ is nonempty for all closed unbounded subsets $C$ of $\alpha$. Let $\omega \lim$ be the class of all ordinals $\alpha$ of cofinality $\omega$, and for any cardinal $\lambda$, let $\omega \lim (\lambda)$ denote the set $(\omega \lim ) \cap \lambda$. Note that for any cardinal $\lambda$ of uncountable cofinality, $\omega \lim (\lambda)$ is a stationary subset of $\lambda$.

The form of Jensen's Diamond Principle used in the paper is stated below, where $E$ is assumed to be a stationary subset of a regular cardinal $\lambda$.

$\diamond(E)$ : There is a sequence $\left\langle S_{\alpha}: \alpha \in E\right\rangle$ such that

(1) $S_{\alpha}$ is a subset of $\alpha$;

(2) for all subsets $X$ of $\lambda,\left\{\alpha \in E: X \cap \alpha=S_{\alpha}\right\}$ is stationary in $\lambda$.

If $\mathrm{GCH}$ holds, then $\diamond(\omega \lim (\lambda))$ holds for all sucessors $\lambda$ of cardinals of uncountable cofinality. In particular, $\diamond\left(\omega \lim \left(w_{m}\right)\right)$ holds for all integers $m \geq 2$. See 1.8, 1.9 and 2.1 of Gregory's [4] paper Higher Souslin trees and the Generalized Continuum Hypothesis for details.

2. A simple example. In this section a counterexample is built for the cartesian product $R=\omega_{2} \times \omega_{1} \times \omega$. This product has order type $\omega \cdot \omega_{1}^{*} \cdot \omega_{2}$ when endowed with lexicographic ordering induced from the usual order on $\omega$ and $\omega_{2}$ 
and the converse of the usual order on $\omega_{1}$. The next definition leads to a characterization of subsets of the product of type $\omega \cdot \omega_{1}^{*} \cdot \omega_{2}$. The alternate terminology is introduced here to produce a suitable analogy for the generalization in $\S 3$. The basic idea is to generalize the definition of cofinal subset of an ordinal to the notion of strongly cofinal subset of a product. The latter definition is made recursively, so preliminaries are in order.

DEFINITION 2.1. (1) In a Cartesian product $X \times Y$, an element $x$ of $X$ occurs with an element $y$ in a subset $A$ of $X \times Y$ if $(x, y)$ is an element of $A$. Given a notion of strongly cofinal subsets of $Y$, an element $x$ of $X$ recurs in a subset $A$ of $X \times Y$, if for a strongly cofinal set of $y, x$ occurs with $y$. Given notions of cofinal subsets of $X$ and strongly cofinal subsets of $Y$, a subset $A$ of $X \times Y$ is strongly cofinal in $X \times Y$ if for a cofinal set of $X$ in $X, x$ recurs in $A$.

(2) To adapt these notions to the product of limit ordinals, $\alpha \times \beta \times \gamma$, one uses the usual notion of cofinal subset of an ordinal both for cofinal where needed above and for strongly cofinal subset of $\gamma$ to initialize the definition.

LEMMA 2.2. Under the lexicographic ordering given above, a subset $S$ of $\omega_{2} \times$ $\omega_{1} \times \omega$ has order type $\omega \cdot \omega_{1}^{*} \cdot \omega_{2}$ if and only if it is strongly cofinal.

THEOREM 2.3. Assume GCH. Then

$$
\omega_{2} \times \omega_{1} \times \omega \nrightarrow(\text { strongly cofinal, infinite path })^{2} .
$$

COROLlary 2.4. Assume GCH. Then

$$
\omega \cdot \omega_{1}^{*} \cdot \omega_{2} \nrightarrow\left(\omega \cdot \omega_{1}^{*} \cdot \omega_{2}, \text { infinite path }\right)^{2} .
$$

As indicated in the introduction, the counterexample is built as the comparability graph of a tree with no infinite branch. If $T=(T,<)$ is a tree, the comparability graph, $G(T)$, is the graph on the vertex set of $T$ in which edges join pairs $\{u, v\}$ for which $u<v$. In [1] the elementary proof of the following fact appears.

LEMMA 2.5 (LEMMA 2.2 OF [1]). If a tree $T=(T,<)$ has no infinite branches, then the comparability graph $G(T)$ has no infinite paths.

A diamond sequence is the starting point of the recursive definition of the tree whose comparability graph is the sought counterexample. The following claim states that there is a diamond sequence suitable for the discussion of strongly cofinal subsets of $\omega_{2} \times \omega_{1} \times \omega$. that

Lemma 2.6. Assume $G C H$. Then there is a sequence $\left\langle U_{\alpha}: \alpha \in \omega \lim \left(\omega_{2}\right)\right\rangle$ so

(1) $U_{\alpha}$ is a subset of $\alpha \times \omega_{1} \times \omega$ strongly cofinal in $\alpha$;

(2) If $A$ is a strongly cofinal subset of $\omega_{2} \times \omega_{1} \times \omega$, then for $\alpha$ in a stationary subset of $\omega \lim \left(\omega_{2}\right), A \cap\left(\alpha \times \omega_{1} \times \omega\right)=U_{\alpha}$.

ProOF. Let $\left\langle S_{\alpha}: \alpha \in \omega \lim \left(\omega_{2}\right)\right\rangle$ be a diamond sequence for $\omega_{2}$. Under the lexicographic order induced by the usual order on all three components, $\omega_{2} \times \omega_{1} \times \omega$ has order type $\omega_{2}$; let $h$ be the order-isomorphism, $h: \omega_{2} \rightarrow \omega_{2} \times \omega_{1} \times \omega$. If $h\left(S_{\alpha}\right)$ is strongly cofinal in $\alpha$, then let $U_{\alpha}=h\left(S_{\alpha}\right)$; otherwise let $U_{\alpha}$ be any set strongly cofinal in $\alpha$ (it is easy to build one). Certainly the sequence so defined has property (1). Note that for all $\alpha$ in a closed unbounded set $C, h(\alpha)=\alpha \times \omega_{1} \times \omega$. Also if $A$ is a strongly cofinal subset of $\omega_{2} \times \omega_{1} \times \omega$, then for $\alpha$ in a closed unbounded set 
$D, A \cap\left(\alpha \times \omega_{1} \times \omega\right)$ is strongly cofinal in $\alpha$. Let $X$ be the subset of $\omega_{2}$ for which $h(X)=A$. If $S$ is a stationary set of $\alpha$ where $X \cap \alpha=S_{\alpha}$, then the intersection of $S$ with $C$ and $D$ is the stationary set required to show property (2) is satisfied.

In the construction of the tree order, to guarantee that no $U_{\alpha}$ extends to a strongly cofinal independent set, one must make many points have predecessors in $U_{\alpha}$. The next definition makes precise a notion of large suitable in this context.

Definition 2.7. A subset $A$ of a limit ordinal $\alpha$ is almost all of $\alpha$ if it is cobounded in $\alpha$. A subset $X$ of a product $\alpha \times \beta$ is almost all of $\alpha \times \beta$ if there is a subset $A$ of $\alpha$ which is almost all of $\alpha$ so that for every $a$ in $A$, the set of $b$ with $(a, b)$ in $X$ is almost all of $\beta$. Recursively, the notion can be extended to larger products.

LEMMA 2.8. If $A$ and $B$ are each almost all of a Cartesian product, then so is $A \cap B$. If $A$ is almost all of a Cartesian product and $C$ is a strongly cofinal subset of it, then their intersection, $A \cap C$, is strongly cofinal.

The proof of Theorem 2.3 continues with two claims.

Claim 2.9. There is an assignment bill: $\omega_{2} \times \omega_{1} \times \omega \rightarrow\left[\omega \lim \left(\omega_{2}\right)\right]^{<\omega}$, which satisfies the following two properties:

(1) if $\delta$ is in $\operatorname{bill}(\alpha, \beta, m)$, then $\delta \leq \alpha$;

(2) if $\delta$ is in $\omega \lim \left(\omega_{2}\right)$ and $\delta \leq \alpha$, then $\delta$ is in $\operatorname{bill}((\alpha) * t)$ for almost all $t$ in $\omega_{1} \times \omega$.

PROOF. One can make such an assignment from enumerations, $e_{\alpha}:|\alpha| \rightarrow \alpha$, which have been extended for notational convenience by the convention $e_{\alpha}(\beta)=$ $e_{\alpha}(0)$ if $\beta>|\alpha|$. If $\delta<\alpha$, then there is some $d<\omega_{1}$ so that $e_{\alpha}(d)=\delta$. For all $\beta>\delta$, there is some $n$ so that $e_{\beta}(n)=d$. That is, $\delta=e_{\alpha}\left(e_{\beta}(n)\right)$. Thus for $s=(\alpha, \beta, m)$, one may set bill $(s)$ to be the intersection of $\omega \lim \left(\omega_{2}\right)$ with the set $\{\alpha\} \cup\left\{e_{\alpha}\left(e_{\beta}(n)\right): n \leq m\right\}$.

ClAIM 2.10. There is a tree order $<_{r}$ on $R=\omega_{2} \times \omega_{1} \times \omega$ which satisfies the following two properties:

(1) if $s=(\alpha, \beta, m)<{ }_{r} t=(\gamma, \delta, n)$, then $\alpha<\gamma$ and $m>n$;

(2) if $\delta$ is in bill $(s)$, then $s$ has a predecessor in the tree in $U_{\delta}$.

PROOF. Let the empty sequence be the root of the tree, and for all finite $\alpha$, let $(\alpha, \beta, m)$ be an immediate sucessor of the root. Next suppose $<_{r}$ has been defined on $\alpha \times \omega_{1} \times \omega$ and fix for a moment a particular point $s=(\alpha, \beta, m)$. To continue the construction, one must determine the immediate predecessor of $s$. First suppose that $\alpha$ is not in $\operatorname{bill}(s)$. Let $\alpha$ be the largest element of bill $(s)$. Then by Claim 2.9, for every $d$ in $\operatorname{bill}(s), d$ is in $\operatorname{bill}(u)$ for almost all $u$ in $\{\alpha\} \times \omega_{1} \times \omega$. Thus there is some $v=(\gamma, \delta, n)$ in $\{\alpha\} \times \omega_{1} \times \omega$ with $n>m$, which has predecessors in $U_{d}$ for all $d$ in $\operatorname{bill}(s)$. Let $v$ be the immediate predecessor of $s$. Now suppose $\alpha$ is in $\operatorname{bill}(s)$. Then $\alpha$ is in $\omega \lim \left(\omega_{2}\right)$, and there is some $a$ less than $\alpha$ so that $a$ recurs in $U_{\alpha}$ and so that $a$ is greater than every element of $\operatorname{bill}(s)$ other than $\alpha$. As before, the set of $u$ in $\{\alpha\} \times \omega_{1} \times \omega$ for which bill $(s) \backslash\{\alpha\}$ is a subset of bill $(u)$, is almost all of $\{\alpha\} \times \omega_{1} \times \omega$. Thus the intersection of $U_{\alpha}$ with this set is strongly cofinal in $\{\alpha\} \times \omega_{1} \times \omega$. Choose some element $v=(\gamma, \delta, n)$ of this set as the immediate predecessor of $s$ with the property that $n>m$. Then $s$ has $v$ as the required predecessor in $U_{\alpha}$ and by the induction hypothesis $s$ has the other required predecessors among the predecessors 
of $v$. Thus the recursive definition of $<_{r}$ can be extended to all points in $\{\alpha\} \times \omega_{1} \times \omega$ in a way that satisfies (1) and (2).

PROOF OF THEOREM 2.3. To prove the theorem, it is enough to check that the tree defined by $<_{r}$ in Claim 2.10 has no infinite branches and no strongly cofinal antichains. Condition (1) of Lemma 2.10 guarantees there is no infinite branch. Condition (2) of Lemma 2.10 is used to show there are no strongly cofinal independent subsets. Suppose $A$ is a strongly cofinal subset of $\omega_{2} \times \omega_{1} \times \omega$. Note that the set of $\alpha<\omega_{2}$ for which $h(\alpha)=\{\alpha\} \times \omega_{1} \times \omega$ and for which $A \cap\left(\{\alpha\} \times \omega_{1} \times \omega\right)$ is strongly cofinal in $\alpha$ is a club. Thus for a stationary set of $\delta$ in $\omega \lim \left(\omega_{2}\right)$, one has $A \cap\left(\{\delta\} \times \omega_{1} \times \omega\right)=U_{\delta}=h\left(S_{\delta}\right)$. Fix one such $\delta$. Choose $\alpha$ larger than $\delta$ so that $\alpha$ recurs in $A$. For almost all $(\gamma, j)$, the ordinal $\delta$ is in $\operatorname{bill}(\alpha, \gamma, j)$. Thus the set of $(\gamma, j)$ for which both $\alpha$ occurs with $(\gamma, j)$ and $\delta$ is in bill $(\alpha, \gamma, j)$ is strongly cofinal in $\omega_{1} \times \omega$. Therefore one may choose $\beta$ and $m$ so that $(\alpha, \beta, m)$ is in $A$ and $\delta$ is in $\operatorname{bill}(\alpha, \beta, m)$. Then $(\alpha, \beta, m)$ and some point of $U_{\delta}$ witness the fact that $A$ contains comparable elements. Thus the tree has no strongly cofinal antichains. Therefore the comparability graph of this tree is the example required to prove Theorem 2.3.

3. A richer example. In this section the previous example is generalized to products of sequences of ordinals. Note that the order type of any ordinal of cardinality $\kappa$ can be represented as the lexicographic order on a set of finite sequences of ordinals less than $\kappa$. Consequences for ordinals of the counterexample of this section are discussed in the following section.

DEFINITION 3.1. For a limit ordinal $\alpha$, let $\operatorname{Seq}(\alpha)$ denote the set $\operatorname{Seq}(\alpha)=$ $\left\{{ }^{m} \alpha: 0<m<\omega\right\}$ of all nonempty finite sequences of ordinals less than $\alpha$. If $s: m \rightarrow \alpha$, then length $(s)=m$ and $\max (s)=\max \{s(0), \ldots, s(m-1)\}$.

DEFINITION 3.2. For each natural number $m$, let

$$
\operatorname{Base}(m)=\operatorname{Seq}\left(\omega_{m}\right) \times \operatorname{Seq}\left(\omega_{m-1}\right) \times \cdots \times \operatorname{Seq}(\omega)
$$

The main counterexample is built on $\operatorname{Base}(m)$. Thus a suitable notion of strongly cofinal must be developed to follow the analogy with the first section. The notion of occurs becomes more interesting. First some notation is introduced to facilitate discussions of initial segments.

NOTATION 3.3. If $s$ is an initial segment of $t$ (or $s=t$ ), then write $s \ll t$. If $s_{i} \ll$ $t_{i}$ for $i=0,1, \ldots, n-1$, then write $s=\left(s_{0}, s_{1}, \ldots, s_{n-1}\right) \ll t=\left(t_{0}, t_{1}, \ldots, t_{n-1}\right)$.

DEFINITION 3.4. A sequence $s=\left(s_{m}, s_{m-1}, \ldots, s_{0}\right)$ from

$$
S=\operatorname{Seq}\left(\alpha_{m}\right) \times \operatorname{Seq}\left(\alpha_{m-1}\right) \times \cdots \times \operatorname{Seq}\left(\alpha_{0}\right)
$$

occurs in a subset $A$ of $S$ if there is some $t=\left(t_{m}, t_{m-1}, \ldots, t_{0}\right)$ in $A$ so that $s \ll t$; in this case one also says that $s_{m}$ occurs with $\left(s_{m}, s_{m-1}, \ldots, s_{0}\right)$ in $A$.

Notice that the concept of occurs may be given a recursive definition. Since the concept of occurs has changed, a corresponding change must be made in the notion of strongly cofinal. Before introducing that concept, a well-ordering on sequences is defined that serves as the foundation for the generalization of almost all to Base $(m)$, as well as being part of the generalization of strongly cofinal.

DEFinition 3.5. For $s, t$ in $\operatorname{Seq}(\alpha), s<_{\text {wo }} t$ if and only if either $\max (s)<$ $\max (t)$, or else $\max (s)=\max (t)$ and length $(s)<\operatorname{length}(t)$, or else $\max (s)=$ $\max (t)$, length $(s)=$ length $(t)$ and $s$ comes before $t$ in the lexicographical ordering. 
LEMMA 3.6. For any limit ordinal $\alpha$, the ordering $<_{\text {wo }}$ on $\operatorname{Seq}(\alpha)$ has the following properties:

(1) if $s$ is a proper initial segment of $t$, then $s<_{\text {wo }} t$;

(2) if $s<_{\text {wo }} t$, then $\max (s) \leq \max (t)$;

(3) a subset $A$ of $\operatorname{Seq}(\alpha)$ is cofinal in $\operatorname{Seq}(\alpha)$ if and only if the set of $\max (s)$ for $s$ in $A$ is cofinal in $\alpha$;

(4) if $\alpha=\kappa$ is a cardinal, then $\operatorname{Seq}(\kappa)$ has order type $\kappa$ under $<_{\text {wo }}$.

DEFinition 3.7. A subset $A$ of $\operatorname{Seq}(\alpha)$ is almost all of $\operatorname{Seq}(\alpha)$ if it is cobounded in $\alpha$. A subset $X$ of a product $\operatorname{Seq}(\alpha) \times \operatorname{Seq}(\beta)$ is almost all of $\operatorname{Seq}(\alpha) \times \operatorname{Seq}(\beta)$ if there is a subset $A$ of $\operatorname{Seq}(\alpha)$ which is almost all of $\operatorname{Seq}(\alpha)$ so that for every $a$ in $A$, the set of $b$ with $(a, b)$ in $X$ is almost all of $\operatorname{Seq}(\beta)$. Recursively, the notion can be extended to larger products.

Notice that the definition of almost all is identical to that of the previous section, from the point of view of the sets with the ordering induced by $<_{\text {wo }}$.

DEFINITION 3.8. A subset of $\operatorname{Seq}(\alpha)$ is strongly cofinal if it is cofinal in the ordering $<_{\text {wo }}$. Extend the definition to products by recursion. Temporarily label $\operatorname{Seq}\left(\alpha_{m}\right) \times \operatorname{Seq}\left(\alpha_{m-1}\right) \times \cdots \times \operatorname{Seq}\left(\alpha_{0}\right)$ by $S$. A sequence $s=\left(s_{m}, s_{m-1}, \ldots, s_{0}\right)$ from $S\left(\alpha_{m}\right)$ recurs in a subset $A$ of $S$ if $s_{m}$ occurs with $v=\left(v_{m-1}, \ldots, v_{0}\right)$ in $A$ for all $v$ in a strongly cofinal subset of $\operatorname{Seq}\left(\alpha_{m-1}\right) \times \cdots \times \operatorname{Seq}\left(\alpha_{0}\right)$. A subset $B$ of $S$ is strongly cofinal in $S$, if there is a cofinal set of sequences $s_{m}$ in $\operatorname{Seq}\left(\alpha_{m}\right)$ which recur in $B$.

LEMMA 3.9. (1) The intersection of two subsets of $S=\operatorname{Seq}\left(\alpha_{m}\right) \times \operatorname{Seq}\left(\alpha_{m-1}\right) \times$ $\cdots \times \operatorname{Seq}\left(\alpha_{0}\right)$ each of which is almost all of $S$ is itself almost all of $S$.

(2) The intersection of a strongly cofinal subset of $S$ and one which is almost all of $S$ is strongly cofinal in $S$.

At this point, by an extension of the arrow notation, the main theorem of the next section can be stated. Namely that theorem says a counterexample can be constructed for Base $(m)$, with strongly cofinal as the notion of large subset.

THEOREM 3.10. Assume GCH. Then for every integer $m \geq 2$, the following relation holds:

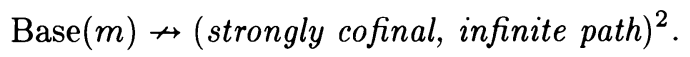

It is convenient to be able to talk about initial segments of $\operatorname{Base}(m)$, where an initial segment $R$ refers to sequences $\left(s_{m}, \ldots, s_{0}\right)$ where every element of $s_{m}$ comes from an initial segment $\beta$ of $\omega_{m}$.

Definition 3.11. Assume $m \geq 2$. If $X$ is a subset of $\operatorname{Base}(m)$ and $\beta$ is an ordinal with $\beta<\omega_{m}$, then $X \mid \beta$ is the set of all $\left(s_{m}, \ldots, s_{0}\right)$ in $X$ with $\max \left(s_{m}\right)<\beta$. To indicate that $X \mid \beta$ is strongly cofinal in $\operatorname{Seq}(\beta) \times \operatorname{Base}(m-1)$, simply write $X \mid \beta$ is strongly cofinal in $\beta$.

LEMMA 3.12. A subset $X$ of $\operatorname{Base}(m)$ is strongly cofinal if and only if the set of $\beta$ for which $X \mid \beta$ is strongly cofinal in $\beta$ contains a closed unbounded set.

PROOF. It follows from the definitions that if the set of $\beta$ for which $X \mid \beta$ is strongly cofinal in $\beta$ contains a closed unbounded set then $X$ is strongly cofinal. So suppose that $X$ is a strongly cofinal subset of $\operatorname{Base}(m)$. If $m=0$, then both concepts reduce to the set $X$ being infinite. So suppose $m>1$. To prove that the 
set of $\beta$ for which $X \mid \beta$ is strongly cofinal in $\beta$ contains a closed unbounded set it suffices to show that given $\delta$ there is such a $\beta$ greater than $\delta$. Suppose $\delta$ is given. By recursion define $\delta_{i}$ and $s_{i}$. First set $\delta_{0}=\delta$. If $\delta_{i}$ has been defined, let $s_{i}$ be an element of $\operatorname{Seq}\left(\omega_{m}\right)$ which recurs in $X$ and has the further property that $\max \left(s_{i}\right)$ is greater than $\delta_{i}$. If $s_{i}$ has been defined, let $S_{i}$ be a subset of $X$ of cardinality $\omega_{m-1}$ of witnesses that $s_{i}$ recurs in $X$. That is, let $S_{i}$ be a subset of $X$ so that $s_{i}$ recurs in $S_{i}$. Let $\delta_{i+1}$ be larger than any ordinal appearing in any sequence $t$ of $\operatorname{Seq}\left(\omega_{m}\right)$ which recurs in $S_{i}$. This $\delta_{i+1}$ can be chosen less than $\omega_{m}$ since it need only be larger than at most $\omega_{m-1}$ ordinals less that $\omega_{m}$. Thus the definition of the sequence continues for countably many steps. Let $\beta$ be the supremum of the $\delta_{i}$ 's. Then $X \mid \beta$ is strongly cofinal in $\beta$.

So far the notion of an initial segment of an ordinal $\alpha$ has been generalized to an initial segment of a subset $X$ of $\operatorname{Base}(m)$ by $X \mid \beta$ and the notion of a subset of $\alpha$ of order type $\alpha$ has been generalized to the notion of strongly cofinal. Next the notion of a diamond sequence on $\omega_{n}$ is extended to Base.

LemMA 3.13. Assume GCH. Then there is a sequence $\left\langle V_{\alpha}: \alpha \in \omega \lim \left(\omega_{m}\right)\right\rangle$, called a diamond sequence suitable for $\operatorname{Base}(m)$, so that

(1) $V_{\alpha}$ is a subset of $\operatorname{Seq}(\alpha) \times \operatorname{Base}(m-1)$ strongly cofinal in $\alpha$;

(2) if $A$ is any strongly cofinal subset of $\operatorname{Base}(m)$, then for $\alpha$ in a stationary subset of $\omega \lim \left(\omega_{m}\right), A \mid \alpha=V_{\alpha}$.

ProOF. Let $\left\langle S_{\alpha}: \alpha \in \omega \lim \left(\omega_{m}\right)\right\rangle$ be a diamond sequence for $\omega_{m}$. Under the lexicographic order induced by $<_{\text {wo }}$ on all components of the product, $\operatorname{Base}(m)$ has order type $\omega_{m}$; let $h$ be the order-isomorphism, $h: \omega_{m} \rightarrow \operatorname{Base}(m)$. If the image under $h$ of $S_{\alpha}$, that is, $h\left(S_{\alpha}\right)$, is strongly cofinal in $\alpha$, then define $V_{\alpha}=h\left(S_{\alpha}\right)$; otherwise let $V_{\alpha}$ be any subset of $\operatorname{Seq}(\alpha) \times \operatorname{Base}(m-1)$ that is strongly cofinal in $\alpha$. Certainly the sequence so defined has property (1). Note that for all $\alpha$ in a closed unbounded set $C, h(\alpha)=\operatorname{Seq}(\alpha) \times \operatorname{Base}(m-1)$. Also if $A$ is a strongly cofinal subset of $\operatorname{Base}(m)$, then for all $\alpha$ in a closed unbounded set $D, A \mid \alpha$ is strongly cofinal in $\alpha$. Let $X$ be the subset of $\omega_{m}$ for which $h(X)=A$. If $S$ is a stationary set of $\alpha$ for which $X \cap \alpha=S_{\alpha}$, then the intersection of $S$ with $C$ and $D$ is the stationary set required to show property (2) is satisfied.

Next the assignment bill is suitably modified to fit with the changes in $\operatorname{Base}(m)$ and in the notion of strongly cofinal.

LEMMA 3.14. There is an assignment bill: $\operatorname{Base}(m) \rightarrow\left[\omega \lim \left(\omega_{m}\right)\right]^{<\omega}$ which satisfies the following three properties:

(1) if $\delta$ is in $\operatorname{bill}\left(s_{m}, \ldots, s_{0}\right)$, then $\delta \leq \max \left(s_{m}\right)$;

(2) if $\delta$ in $\omega \lim \left(\omega_{m}\right)$ is less than $\max \left(s_{m}\right)$, then $\delta$ is in $\operatorname{bill}\left(\left(s_{m}\right) * t\right)$ for almost all $t$ in $\operatorname{Base}(m-1)$;

(3) if $s \ll t$, then bill $(s)$ is a subset of $\operatorname{bill}(t)$.

PROOF. The proof of this lemma is very much like the analogous lemma of the previous section where the required function was defined largely by cardinality considerations. The same approach can be used here, although the basic trick with enumerations may need to be done up to $m$ times. Such an approach would give a first approximation, invoice, to the function desired here, one which would satisfy the first two conditions of the lemma. To adjust it to satisfy the last condition of 
the lemma, one would need to define bill $(t)$ to be the union of invoice(s) for all $s$ with $s \ll t$.

LEMMA 3.15. Assume bill is defined as in the previous lemma. Then there is a tree order $<_{t}$ on $\operatorname{Base}(m)$ which satisfies the following two properties:

(1) if $s=\left(s_{m}, s_{m-1}, \ldots, s_{0}\right)<_{t} t=\left(t_{m}, t_{m-1}, \ldots, t_{0}\right)$, then $s_{m}<_{\text {wo }} t_{m}$ and $s_{0}>_{\text {wo }} t_{0}$

(2) if $\delta$ is in bill $(s)$, then some $u$ in $V_{\delta}$ is a predecessor of $s$ in the tree order.

PROOF. The definition of the tree order is by recursion on $\operatorname{Seq}\left(\omega_{m}\right)$ under the ordering $<_{\text {wo }}$. Let the empty sequence be the root of the tree, and for all $s=\left(s_{m}, s_{m-1}, \ldots, s_{0}\right)$ with $\max \left(s_{m}\right)$ finite, let $s$ be an immediate sucessor of the root.

Next suppose that for some $u_{m}$ with $\max \left(u_{m}\right)$ infinite, the tree order $<_{t}$ has been defined successfully for all $t=\left(t_{m}, t_{m-1}, \ldots, t_{0}\right)$ with $t_{m}<_{\text {wo }} u_{m}$. Fix for a moment a particular point $s=\left(s_{m}, s_{m-1}, \ldots, s_{0}\right)$ with $s_{m}=u_{m}$. To continue the construction, one must determine the immediate predecessor of $s$. First suppose that $\alpha=\max \left(s_{m}\right)$ is not in $\operatorname{bill}(s)$. Let $\beta$ be the largest element of $\operatorname{bill}(s)$. Then by Lemma 3.14 , for every $\delta$ in $\operatorname{bill}(s), \delta$ is in $\operatorname{bill}((\beta) * w)$ for almost all $w$ in $\operatorname{Base}(m-1)$. Thus by the inductive hypothesis, there is some $\left(v_{m}, v_{m-1}, \ldots, v_{0}\right)$ in $\{(\beta)\} \times \operatorname{Base}(m-1)$ with $v_{0}>_{\text {wo }} s_{0}$, which has predecesors in $V_{\delta}$ for all $\delta$ in bill $(s)$. Let $v$ be the immediate predecessor of $s$.

Now suppose $\alpha$ is in $\operatorname{bill}(s)$. Then $\alpha$ is in $\omega \lim \left(\omega_{m}\right)$ and $V_{\alpha}$ is strongly cofinal in $\alpha$. Thus there is a sequence $r$ in $\operatorname{Seq}(\alpha)$ which recurs in $V_{\alpha}$ and which has the property that $\max (r)=\beta$ for some $\beta$ greater than every element of $\operatorname{bill}(s)$ other than $\alpha$. Let $R$ be the set of all $w$ in $\operatorname{Base}(m-1)$ so that $r$ occurs with $w$ in $V_{\alpha}$. Let $W$ be the set of $w$ in $\operatorname{Base}(m-1)$ for which $\operatorname{bill}(s) \backslash\{\alpha\}$ is a subset of $\operatorname{bill}(r * w)$. Since $W$ is almost all of $\operatorname{Base}(m-1)$, the intersection of $R$ and $W$ is strongly cofinal in $\operatorname{Base}(m-1)$. Choose some element $w=\left(w_{m-1}, w_{m-2}, \ldots, w_{0}\right)$ of this set with the property that $w_{0}>_{\text {wo }} s_{0}$. Since $r$ occurs with $w$ in $V_{\alpha}$, there is an element $v=\left(v_{m}, v_{m-1}, \ldots, v_{0}\right)$ of $V_{\alpha}$ which witnesses this fact. Let $v$ be the immediate predecessor of $s$. Since $v$ is in $V_{\alpha}, \max \left(v_{m}\right)$ is less than $\alpha$, so $v_{m}<_{\text {wo }} s_{m}$. By the definition of occurs, $r * w \ll v$, so in particular $w_{0}<_{\text {wo }} v_{o}$. Consequently, $v_{0}>_{\text {wo }} s_{0}$ and the pair $s, v$ satisfies condition (1). By transitivity, the pairings of $s$ with predecessors of $v$ also satisfy condition (1). Recall that $w$ was chosen so that $\operatorname{bill}(s) \backslash\{\alpha\}$ is a subset of $\operatorname{bill}(r * w)$. Since $r * w \ll v$, by condition (3) of Lemma 3.14, $\operatorname{bill}(s) \backslash\{\alpha\}$ is also a subset of bill $(v)$. Thus $v$ is the required predecessor of $s$ in $V_{\alpha}$, and by the induction hypothesis, $v$ has among its predecessors the other required predecessors of $s$.

Therefore the definition of $<_{t}$ can be extended to all points in $\left\{u_{m}\right\} \times \operatorname{Base}(m-1)$ in a way that satisfies (1) and (2). Thus by recursion on $\operatorname{Seq}\left(\omega_{m}\right)$ under the order $<_{\text {wo }}$, the definition of the tree order can be extended to all of Base $(m)$.

PROOF OF THEOREM 3.10. Consider the comparability graph of the tree order of Lemma 3.15. Condition (1) of that lemma together with Lemma 3.14 guarantee that the graph has no infinite path. Condition (2) of Lemma 3.15 is used to show there are no strongly cofinal independent subsets. Suppose $A$ is a strongly cofinal subset of $\operatorname{Base}(m)$. By Lemma 3.13, there is some $\alpha$ in $\omega \lim \left(\omega_{m}\right)$ so that $A \mid \alpha=V_{\alpha}$. Since $A$ is strongly cofinal, there is some $r$ in $\operatorname{Seq}\left(\omega_{m}\right)$ with 
$\max (r)>\alpha$ so that $r$ recurs in $A$. For almost all $w$ in $\operatorname{Base}(m-1)$, the ordinal is in $\operatorname{bill}(r * w)$. Thus for some particular $w$ in Base $(m-1), r$ occurs with $w$ in $A$ and $\alpha$ is in $\operatorname{bill}(r * w)$. Let $t$ witness that $r$ occurs with $w$ in $A$. Since $r * w \ll t$, the ordinal $\alpha$ is also in bill $(t)$. Thus among the predecessors of $t$ in the tree is an element $v$ of $V_{\alpha}$. The two points $v$ and $t$ show that $A$ is not independent. Thus the comparability graph is the desired graph to prove Theorem 3.10.

4. Pinning the example to ordinals. In this section the result of the previous sections are applied to the ordinals to get a variety of results.

The simple product of $\S 2$ was generalized in $\S 3$ to a product of finite sequences of ordinals. In the simple case one could describe the large subsets of interest by an order type induced on the product via a lexicographic ordering where alternate terms received the usual or the converse of the usual well-order. One can of course expand this concept to products of more than the three sets of $\S 2$. In this case, if one by an abuse of notation identifies an ordinal $\alpha$ with the sequence $(\alpha)$, then the large subsets of such a product, $\omega_{m} \times \omega_{m-1} \times \cdots \times \omega$, are strongly cofinal in $\operatorname{Base}(m)$. Thus the generalization to finite sequences includes the simpler products.

T. Carlson [2] has used such products in his paper on pinning. He has studied the same order structure in terms of ideals and in terms of regularly branching trees. In order to preserve infinite paths, one-to-one pinning maps are required.

DEFINITION 4.1. Suppose we are given sets $S, T$ together with notions of large subsets of $S$ and $T$. Then $S$ can be pinned to $T$, in notation, $S \rightarrow T$, if there is a function $p: S \rightarrow T$ so that whenever $A$ is a large subset of $S$, then the image, $B=p(A)$, is a large subset of $T$. In this case, the function $p$ is called a pinning map.

The concept of pinning was introduced to transfer pinning relations from one ordinal to another, so the notion of large originally used was that of a subset of the full order type. Carlson generalized it by looking at ideals as giving small sets and using sets not in the ideal for large in the sense mentioned above. For the products that have been discussed in this paper, the notion of large is strongly cofinal. One of Carlson's results is that for every positive integer $m$, there is an ordinal $\alpha$ between $\omega_{m-1}$ and $\omega_{m}$ so that $\alpha$ can be pinned to $\omega_{m} \times \omega_{m-1} \times \cdots \times \omega$. Since these sets can be identified with strongly cofinal subsets of $\operatorname{Base}(m)$, Carlson's result may be recast as follows.

LEMMA 4.2. (See Lemma 3, p. 46 and Lemma 6, p. 61 of [2].) If $m$ is a positive integer, then there are ordinals $\alpha$ between $\omega_{m}$ and $\omega_{m+1}$ so that $\alpha \rightarrow \operatorname{Base}(m)$. Furthermore the pinning mapping may be chosen so that it is one-to-one.

To prove this result, Carlson looks at ordinals that are products of smaller ordinals that are sufficiently independent to allow mappings of each factor to be combined to a mapping of the product. Note $\beta \cdot \alpha$ can always be pinned onto $\alpha$. Thus for each $m$, there are cofinally many ordinals below $\omega_{m+1}$ which can be pinned onto Base $(m)$. These ordinals satisfy a negative partition relation as a corollary to the result about $\operatorname{Base}(m)$ of the previous section, since if $S$ can be pinned to $T$ and $T$ satisfies a negative relation, then so does $S$. 
THEOREM 4.3. Assume GCH. Let $m$ be a positive integer with $m \geq 2$. Then for a cofinal set of $\alpha<\omega_{m+1}$,

$$
\alpha \nrightarrow(\alpha, \text { infinite path })^{2} .
$$

Carlson has as his goal finding an ordinal which can be pinned to larger ordinals. He has isolated the heart of his argument on p. 58 of his article. There he states that for a given cardinal $\kappa$ and a given notion of a large subset of $S$, if $\kappa^{<\kappa}=\kappa$ and there is a function $\phi: S \rightarrow[\kappa]^{<\omega}$ so that $\bigcup \phi(A)=\kappa$ for all large subsets $A$ of $S$, then $S$ can be pinned to cofinally many ordinals below $\kappa^{+}$. He also shows how to use the function $\phi$ to pin $S$ to all regular cardinals less than or equal to $\kappa$. The definitions of Base $(m)$ and strongly cofinal are designed for the task that Carlson set for himself, since the function bill defined in $\S 3$ has exactly the required property. What is not so clear is which ordinals can be pinned to Base $(m)$. Baumgartner and Larson [1] have proved the following result for $m=1$.

LEMMA 4.4. (See Lemma 5.1 of [1].) If $\alpha$ is an ordinal between $\omega_{1}$ and $\omega_{2}$, then there is a one-to-one pinning map from $\alpha$ into Base(1).

This result combined with the work of Carlson shows that every ordinal $\alpha$ between $\omega_{1}^{\omega+2}$ and $\omega_{2}$ can be pinned to cofinally many ordinals less than $\omega_{2}$.

For larger ordinals the situation is not so simple. As indicated above, Carlson shows how given a positive integer $m$, to get an ordinal $\alpha_{m}$ that can be pinned onto $\operatorname{Base}(m)$. However, as Carlson indicates, it is also not difficult to pin $\operatorname{Base}(m)$ onto $\omega_{n}$ for $n \leq m$. Furthermore, Carlson (see Lemma 4 on p. 60 of [2]) has shown that assuming GCH, for all $n$ with $0<n<m$, there are cofinally many ordinals below $\omega_{m+1}$ which do not pin to $\omega_{n}$. Since $\operatorname{Base}(m)$ can be pinned to $\omega_{n}$ for all $n \leq m$, it follows that cofinally many ordinals below $\omega_{m+1}$ cannot be pinned to $\operatorname{Base}(m)$.

Thus the counterexample on $\operatorname{Base}(m)$ has limitations. The consequences for ordinals listed so far are all ones that follow from counterexamples on the product $\omega_{m} \times \omega_{m-1} \times \cdots \times \omega$, or its modification to $\left(\omega_{m} \times \omega_{m-1} \times \cdots \times \omega\right) \times \omega_{m}$ for one-toone pinning maps. The ordinals $\omega_{2}^{\omega_{1} \omega+2}$ and $\omega_{2}^{\omega_{1}+\omega}$ are examples of ordinals that one can pin without great difficulty onto $\operatorname{Base}(m)$, while whether or not one can pin them onto the above-mentioned product is not so clear. Indeed, the question of exactly which ordinals can be pinned, in a one-to-one fashion, to $\operatorname{Base}(m)$, is a question which the current paper does not address.

Other questions immediately present themselves. One of the ones closest to the original motivation for the paper is the following.

QUESTION 4.5. Assume GCH. Is there an ordinal $\alpha$ between $\omega_{2}^{\omega_{1}+2}$ and $\omega_{3}$ so that $\alpha \rightarrow(\alpha \text {, infinite path })^{2}$ ?

As mentioned in the introduction, the methods of this paper give no information about ordinals larger than $\omega_{\omega}$. Instead, the methods of the paper suggest that products might be interesting to look at. The original paper of Erdös, Hajnal and Milner [3] already answered some of the most attractive questions for simple products without requiring any additional hypothesis, since these products give rise to ordinal order types. In particular, $\omega \times \omega_{2}, \omega_{1} \times \omega_{2}$, and $\omega \times \omega_{1} \times \omega_{2}$ all satisfy the positive relation. In the same paper, the three authors show that $\omega_{2}^{\omega+1} \cdot \omega_{1}$ has the positive relation. Since this ordinal can be pinned to $\omega_{1} \times \omega \times \omega_{2}$, the latter must also have the positive relation. A cardinality argument can be used to show that 
under the assumption of GCH, $\omega_{2} \times \omega$ also has the positive relation. In $\S 2$ of this paper, the proof that $\omega_{2} \times \omega$ has the negative relation appears. Simple products not yet decided are $\omega_{2} \times \omega_{1}, \omega \times \omega_{2} \times \omega_{1}$, and $\omega_{1} \times \omega_{2} \times \omega$.

\section{REFERENCES}

1. J. Baumgartner and J. Larson, A diamond example of an ordinal graph with no infinite paths.

2. T. Carlson, The pin-up conjecture, Axiomatic Set Theory, Contemp. Math., vol. 31, Amer. Math. Soc., Providence, R.I., 1984, pp. 41-62.

3. P. Erdös, A. Hajnal and E. C. Milner, Set mappings and polarized partition relations, Combinatorial Theory and its Applications, Balatonfüred, Colloq. Math. Soc. János Bolyai, vol. 4, North-Holland, Amsterdam, 1969, pp. 327-363.

4. J. Gregory, Higher Souslin trees and the Generalized Continuum Hypothesis, J. Symbolic Logic 41 (1976), 663-671.

5. T. Jech, Set theory, Academic Press, New York, 1978.

6. J. Larson, Martin's Axiom and ordinal graphs: large independent sets or infinite paths.

Department of Mathematics, University of Florida, Gainesville, Florida 32611 